\title{
La educación para el desarrollo y los derechos de la infancia: el papel de las agencias internacionales y el impacto de la formación en la transformación de los contextos
}

\author{
M. a Ángeles Espinosa Bayal \\ Universidad Autónoma de Madrid. España. \\ mangeles.espinosa@uam.es
}

Almudena Martínez Gimeno
Ricardo García Pérez
Universidad Pablo de Olavide. España.
amartinez@upo.es
rgarper@upo.es

Recibido: 1/5/2019

Aceptado: 30/9/2019

Publicado: 20/7/2020

\section{Resumen}

El objetivo del artículo es describir el papel que las agencias internacionales tienen en la educación para el desarrollo desde un enfoque de derechos de la infancia. Considerando, como uno de los principales hitos, la aprobación en 1989 de la Convención de las Naciones Unidas sobre los Derechos del Niño y estableciendo el bienestar infantil como base para la consecución de la Agenda 2030, se detalla una experiencia exitosa, el Programa Ciudades Amigas de la Infancia de UNICEF-Comité Español, y la evaluación del impacto del Programa de Formación de Postgrado en Derechos y Políticas Municipales de Infancia. Los resultados ponen de manifiesto que la formación mejora la estabilidad laboral, las competencias profesionales y el desarrollo de iniciativas integrales de infancia, pero también que no tiene un efecto tan positivo en el reconocimiento que las organizaciones otorgan a los profesionales. Se concluye acerca de la importancia de la formación como una potente herramienta en la transformación de los contextos socioeducativos.

Palabras clave: bienestar infantil; evaluación de impacto; índice de desarrollo humano; formación en derechos de infancia

Resum. L'educació per al desenvolupament $i$ els drets de la infancia: el paper de les agències internacionals $i$ l'impacte de la formació en la transformació dels contextos

L'objectiu de l'article és descriure el paper de les agències internacionals en l'educació per al desenvolupament des d'un enfocament de drets de la infancia. Considerant una de les principals fites l'aprovació el 1989 de la Convenció de les Nacions Unides sobre els Drets de l'Infant i establint el benestar infantil com a base per a l'assoliment de l'Agenda 2030, s'hi detalla una experiència d'èxit, com és el cas del Programa Ciutats Amigues de la Infancia d'UNICEF-Comitè Espanyol, i l'avaluació d'impacte del Programa de Formació de Postgrau en Drets i Polítiques Municipals d'Infancia. Els resultats posen de manifest que 
la formació millora l'estabilitat laboral, les competències professionals i el desenvolupament d'iniciatives integrals d'infancia, però també que no té un efecte tan positiu en el reconeixement que les organitzacions atorguen als professionals. Es conclou sobre la importància de la formació com una eina potent en la transformació dels contextos socioeducatius.

Paraules clau: benestar infantil; avaluació d'impacte; índex de desenvolupament humà; formació en drets d'infancia

Abstract. Education for development and children's rights: The role of international agencies and the impact of training on the transformation of contexts

The aim of the article is to examine the role of international agencies in education for development from the perspective of children's rights. We consider the adoption of the United Nations Convention on the Rights of the Child in 1989 as one of the main milestones and child welfare as a cornerstone to achieve the 2030 Agenda. The successful experience of the UNICEF Child Friendly Cities Initiative of the Spanish Committee and the impact of the Master's Degree in Municipal Rights and Policies for Children are described. The results show that training improves job stability, professional skills and the development of comprehensive childhood initiatives, but does not have such a positive effect on organizations' recognition of professionals. It is concluded that education is a powerful tool for the transformation of socioeducational contexts.

Keywords: child welfare; impact evaluation; human development index; children's rights training

\section{Sumario}

1. Introducción

2. El papel de las agencias internacionales en el desarrollo del enfoque de los derechos de la infancia

3. La estrategia de educación para el desarrollo y los derechos de la infancia en España
4. Una experiencia exitosa: el programa "Ciudades amigas de la infancia»

\section{Conclusiones}

Referencias bibliográficas

\section{Introducción}

La Agenda 2030 compromete a todos los países a alcanzar los objetivos de desarrollo sostenible (ODS) para poner fin a la pobreza, proteger el planeta y garantizar que las personas vivan en entornos seguros y prósperos. Estos objetivos sustituyen a los objetivos de desarrollo del milenio (ODM), que impulsaron el progreso en esferas como la reducción de la pobreza económica; el acceso al agua y al saneamiento; la disminución de la mortalidad infantil y la mejora de la salud materna; el aumento de las tasas de escolarización primaria, y los avances en la lucha contra el VIH/SIDA y otras enfermedades, como la malaria o la tuberculosis. 
Estos logros no se habrían alcanzado de no ser por la transición en el concepto de desarrollo desde una definición basada en términos macroeconómicos a un nuevo concepto que lo define en términos de desarrollo humano. Este nuevo enfoque deriva del informe elaborado por Amartya Senn para el Programa de Naciones Unidas para el Desarrollo (PNUD), en el que el desarrollo se define como un proceso que amplía las oportunidades del ser humano, pudiendo llegar a ser infinitas y cambiantes en el tiempo siempre que se produzcan tres condiciones básicas: disfrutar de una vida prolongada y saludable, adquirir conocimientos y tener acceso a los recursos necesarios para lograr un nivel de vida digno (PNUD, 1990). Esta nueva conceptualización empodera a las personas para que reclamen los derechos recogidos en los tratados internacionales, lo que supone que dichos tratados, en general, y la Declaración Universal de Derechos Humanos, en particular, se convierten en herramientas fundamentales para conseguir la justicia social.

En 1997, el Programa de Reforma de la ONU hace un llamamiento a las organizaciones del Sistema de Naciones Unidas para que el enfoque de derechos humanos (DH) sea incluido en todos sus mandatos. Desde entonces, muchas de ellas han adoptado el enfoque de $\mathrm{DH}$ y de cooperación al desarrollo, tal como consta en el Statement on a Common Understanding of Human Rigths-Based Approach to Development Cooperation, elaborado por el PNUD (UNGD Human Rights Working Group, 2003). Así, los DH se convierten en un eje transversal de la educación para el desarrollo (EpD), entendiéndose que, en caso de vulneración, no es posible acceder a los derechos de ciudadanía, que son realmente los que sirven a su fundamentación. La EpD supone un proceso a partir del cual se generan conciencias críticas con el objetivo de que la ciudadanía participe de manera activa y responsable en la toma de decisiones políticas, económicas y sociales, para construir una sociedad más comprometida con la solidaridad y la justicia social, en la que se hagan realidad los DH de todas las personas (CONGD, 2005; Ministerio de Asuntos Exteriores y de Cooperación, 2008).

Todo lo dicho como fundamento de la EpD podría aplicarse a los derechos de la infancia, derechos que la comunidad internacional reconoce a todos los niños, niñas y adolescentes (NNA), con independencia de cualquier otra consideración, y que aparecen recogidos en la Convención de Naciones Unidas sobre los Derechos del Niño (CDN), aprobada en 1989. La Agenda 2030 solo será posible si somos capaces de garantizar el bienestar de los NNA permitiéndoles participar en la toma de las decisiones que les afectan, ejerciendo así de manera plena sus derechos de ciudadanía. Hacer realidad los ODS es una apuesta por un modelo social y político que sitúa a la infancia como prioridad, especialmente a aquella que se encuentra en situación de mayor vulnerabilidad (UNICEF, 2017).

Dedicaremos las páginas siguientes a analizar el papel que las agencias internacionales han jugado en la inclusión del enfoque de derechos de la infancia en la EpD, presentando una experiencia liderada por UNICEF Comité Español como ejemplo de las iniciativas que actualmente se desarrollan. 


\section{El papel de las agencias internacionales en el desarrollo del enfoque de los derechos de la infancia}

La década de 1990 vendrá marcada por dos hitos fundamentales en relación con la introducción de la perspectiva de los derechos de la infancia en la EpD. El primero fue la aprobación de la CDN, el tratado internacional jurídicamente vinculante más ampliamente ratificado que reconoce derechos económicos, sociales, culturales, civiles y políticos a los NNA, al mismo tiempo que señala las obligaciones y las responsabilidades de los adultos que tienen a su cargo el cuidado y la protección de los menores y, en su defecto, el papel subsidiario que debe ejercer el Estado. La Convención considera al niño como sujeto activo de derechos. El papel activo que los NNA pueden adoptar en la construcción de su propio desarrollo, así como la importancia que se da a la participación infantil, hacen que puedan convertirse en sujetos críticos dispuestos a ejercer todos y cada uno de sus derechos y, por tanto, en poseedores de una ciudadanía global que ayude a construir un mundo más justo y equitativo. La Convención como herramienta para la protección y la garantía de derechos de la infancia se ha visto posteriormente ampliada, tanto con sus tres protocolos facultativos - el relativo a la participación de los niños en conflictos armados y el relativo a la venta de niños, la prostitución infantil y la utilización de NNA en la pornografía, ambos aprobados en el 2000, y el relativo al procedimiento de comunicaciones, aprobado en 2011- como con las 21 observaciones generales elaboradas por el Comité de Derechos del Niño, más dos elaboradas en colaboración con el Comité de Protección de los Derechos de Todos los Trabajadores Migratorios y sus Familias, para ayudar a la adecuada interpretación y aplicación de los derechos recogidos en la Convención.

Aunque la aprobación de la Convención marca un hito, anteriormente ya se habían adoptado algunas iniciativas encaminadas a garantizar los derechos de los NNA, de las cuales destacan la Convención sobre el Consentimiento para el Matrimonio, la Edad Mínima para Contraer Matrimonio y el Registro de los Matrimonios, aprobada en 1964, que en su artículo 2 establece que los estados parte adoptarán medidas legislativas para determinar la edad mínima para contraer matrimonio. El Convenio n. ${ }^{\circ} 138$ de la OIT sobre la edad mínima de admisión al empleo, aprobado en 1973, que en el apartado 3 del artículo 2 establece que la edad mínima para trabajar no será inferior a la edad en la que acabe la escolarización obligatoria, y en todo caso, a los 15 años, y el Convenio sobre la Eliminación de Todas las Formas de Discriminación contra la Mujer, aprobado en 1979, que en el apartado 2 del artículo 16 señala que no tendrán efecto jurídico los esponsales ni el matrimonio de niños y que se fijará una edad mínima para su celebración. Entre las iniciativas surgidas después de la aprobación de la Convención cabría señalar el Convenio n. ${ }^{\circ} 182$ de la OIT sobre las Peores Formas de Trabajo Infantil (1999), que establece la necesidad de eliminar de forma inmediata la esclavitud, el reclutamiento para participar en conflictos armados, la prostitución o la producción pornográfica, el tráfico de estupefacientes y el trabajo que dañe la 
salud física o psicológica de los niños, además de la Convención sobre los Derechos de las Personas con Discapacidad, aprobada en 2006, que señala en el apartado 1 del artículo 24 que los estados deben velar porque los niños con discapacidad tengan acceso a la educación primaria y secundaria en igualdad de oportunidades.

El segundo hito fue la publicación, por parte de UNICEF, del informe Ajuste con rostro humano, en el que se inicia un planteamiento que posteriormente adoptará el PNUD, que supera el enfoque economicista de desarrollo para centrarse en un enfoque de DH (Cornia y Jolly, 1987). A partir de este trabajo se define el índice de desarrollo humano (IHD), indicador sintético que combina tres dimensiones para que una persona pueda alcanzar el máximo de sus posibilidades: salud —-medida según la esperanza de vida al nacer-; educación - medida por la tasa de alfabetización de los adultos y la tasa bruta combinada de matriculación en educación primaria, secundaria y superior, así como la duración de la educación obligatoria—, y riqueza — medida por el PIB per cápita en dólares internacionales- (PNUD, 1990). Este enfoque entiende el desarrollo como un proceso de expansión de las libertades que permite a las personas desarrollar en sus propios entornos iniciativas beneficiosas para ellas y para quienes les rodean, siendo capaces de generar cambios encaminados a la construcción de sociedades más libres, democráticas y equitativas (Sen, 2000). Como consecuencia del papel preponderante de los derechos humanos, los derechos de la infancia y la EpD, se organizó, en el año 2000, la Cumbre Mundial a Favor de la Infancia, en la que 60 estados se comprometieron a mejorar las condiciones de vida para los NNA y ratificaron la Declaración del Milenio, en la que aparece el firme compromiso de dar prioridad a su protección para garantizar el progreso de la humanidad. En esta cumbre se fijaron los ODM ratificados en 2005 y se estableció como fecha límite para su consecución el año 2015. Posteriormente, en 2012, tras monitorizarlos y comprobando que aún quedaban importantes retos pendientes (UNICEF, 2010), se celebra la Conferencia de Naciones Unidas sobre el Desarrollo Sostenible, donde se gestan los ODS aprobados en 2015 e incluidos en la Agenda 2030, que suponen una oportunidad única para no dejar a ningún niño atrás, aunque su consecución necesita el trabajo conjunto de todos y cada uno de los países, de los gobiernos y de los representantes del sector privado, de la sociedad civil y de la ciudadanía.

\section{La estrategia de educación para el desarrollo y los derechos de la infancia en España}

España ratificó la CDN en diciembre de 1989 y entró en vigor en enero de 1990, lo que supuso un cambio importante en el trabajo que se desarrollaba en $\mathrm{EpD}$, ya que se introdujo el enfoque de derechos de la infancia. Un primer intento de aproximación a la $\mathrm{EpD}$ desde este enfoque es el que hace la, entonces, Asociación UNICEF-España publicando el libro Educación para el desarrollo (UNICEF, 1978), en el que se aportaban materiales para trabajar con el 
alumnado acerca de las temáticas de salud, pobreza, agua y desnutrición. En 1993 se crea el Departamento de Educación para el Desarrollo y Programas de UNICEF Comité Español (UCE), que prioriza el trabajo en EpD desde el enfoque de derechos con el objetivo de que estos se conviertan en "principios éticos y perdurables» que guíen las actuaciones a nivel autonómico, nacional e internacional, anticipándose así a la misión que la Asamblea General de Naciones Unidas encomienda a UNICEF en 1996, por la que le confía el mandato de promover la protección de los derechos del niño, ayudar a satisfacer sus necesidades básicas y aumentar sus oportunidades, para que alcance sus potencialidades al máximo (UNICEF, 1996a).

Paralelamente y en colaboración con la Fundación de Cooperación y Desarrollo (FUNCOE), posteriormente integrada en Save the Children, se pone en marcha en 1993 el programa "Solidaridad con la infancia latinoamericana", con una propuesta articulada en tres fases: conocer los problemas de la infancia y sus causas, comunicarlos a la sociedad y establecer hermanamientos con centros educativos de países en vías de desarrollo. Para reforzar el programa, en 1995 se edita en lengua quechua la revista Jatun Sunqu (Corazón Grande), acompañada de una guía didáctica que se distribuye a los centros educativos para trabajar la educación en valores con el alumnado de 12 a 14 años. La rápida implantación del programa supuso la creación de un equipo profesional en UNICEF-Comité Español formado en la CDN y comprometido con su implementación, cuya labor se vio fortalecida por la publicación de un manual que abordaba diferentes temáticas sobre derechos de la infancia, entre otros: igualdad de género; niños soldados; la ciudad y los niños, y agua, saneamiento, higiene y salud (UNICEF, 1996a).

Al mismo tiempo, a iniciativa de UNICEF Ginebra y algunos comités nacionales, surge el Grupo del Mediterráneo para trabajar con jóvenes de diferentes culturas desde un enfoque de $\mathrm{EpD}$ asumiendo valores como la solidaridad, la interculturalidad, el respeto y la tolerancia y el cuidado del medio ambiente. En 1997 se transforma en el programa «Paz en el Mediterráneo», que, con el apoyo de la Agencia Española de Cooperación Internacional (AECI), trabaja los conceptos clave de la EpD: interdependencia; justicia social; imágenes y percepciones; conflictos y su solución, y cambios y futuro (Fontaine, 1995). Posteriormente se generaron alianzas estratégicas con diferentes sectores para ampliar la magnitud del enfoque de derechos de la infancia en la sociedad española.

Las alianzas entre el UCE y el ámbito municipal surgen en 1992 con Alcaldes Defensores de los Niños, con lo que se resalta la importancia del ámbito municipal en la protección, la promoción y la garantía de los derechos de los NNA. Esta iniciativa se ve potenciada, en el año 1996 en Estambul, por la Conferencia de Naciones Unidas sobre Asentamientos Urbanos Hábitat II. Se constituye la Secretaría Permanente del programa "Ciudades amigas de la infancia» (CAI), que explicaremos en el siguiente apartado de este artículo, formada por el Ministerio de Sanidad, Consumo y Bienestar Social, la Federación Española de Municipios y Provincias (FEMP), el UCE y el Instituto 
Universitario IUNDIA, que se encarga de la supervisión y el control del programa y de los criterios para el acceso al reconocimiento de una CAI.

Para el UCE la participación infantil (artículo 12, CDN) es un elemento esencial del enfoque de derechos de la infancia en la $\mathrm{EpD}$ que se viene trabajando desde 1998, cuando el Comité del País Vasco pone en marcha la iniciativa La Voz de la Infancia, configurada como un proceso electoral real dirigido a 80.000 NNA de 8 a 11 años, que debían elegir uno entre 12 derechos propuestos. Con más de 25.000 participantes, marca la participación como herramienta para la construcción de ciudadanía, mecanismo esencial de la EpD. Desde entonces el UCE ha seguido enfatizando la importancia de escuchar a los NNA, y actualmente existen consejos de participación infantil en todos los municipios que tienen el reconocimiento CAI. Este derecho también se trabaja en muchos centros educativos que participan en el programa «Educación en derechos» (UNICEF, 2018b).

En 1997, el UCE establece una colaboración más amplia con la Universidad Autónoma de Madrid (UAM) coorganizando el máster en Necesidades y Derechos de la Infancia y la Adolescencia, título propio de la UAM, actualmente denominado máster en Necesidades, Derechos y Cooperación al Desarrollo en la Infancia, cuyo objetivo es formar profesionales altamente cualificados y expertos en el diseño, el desarrollo y la implementación de políticas de infancia desde el enfoque de derechos. La colaboración con la UAM se amplía en 1999, al crear el Instituto Universitario UAM-UNICEF de Necesidades y Derechos de la Infancia y la Adolescencia (IUNDIA), y años más tarde, en 2011, con la constitución de la Red de Universidades por los Derechos de la Infancia y la Adolescencia (RUNDIA), cuyo objetivo es desarrollar investigaciones, publicaciones y formación de postgrado en materia de derechos de la infancia, así como la puesta en marcha de actividades de EpD desde el enfoque de derechos y el fomento de la cooperación en el ámbito universitario.

La alianza con las administraciones públicas incluye, además del Programa CAI, el apoyo al programa "Enrédate con UNICEF», que se presentó en el año 2000 como alternativa al programa "Juventud solidaria" y que contaba con una página web para mantener el contacto con los centros y los materiales para trabajar la EpD con el alumnado, el profesorado y las familias, disponible en las lenguas cooficiales del Estado. Recientemente se ha reconvertido en el programa "Educación en derechos», que está financiado por la AECID e incorpora la formación del profesorado en materia de derechos de la infancia como elemento esencial.

\section{Una experiencia exitosa: el programa "Ciudades amigas de la infancia»}

El programa CAI se implementa en España en 2001 y desde entonces ha tenido un crecimiento exponencial (UNICEF, 2007). Actualmente 274 municipios están reconocidos como CAI por diseñar políticas municipales que favorecen el bienestar de los NNA garantizando el cumplimiento de sus derechos y disponiendo, además, de un órgano de participación infantil (UNICEF, 
2018b). El 41\% de la población española menor de 18 años vive en un municipio amigo de la infancia. El reconocimiento CAI supone un proceso de control y supervisión de la Secretaría Técnica del Programa que implica la necesidad de desarrollar políticas integrales de infancia en las que participen todas las concejalías mediante mecanismos de comunicación estables para la toma de decisiones.

El diseño y la implementación de una política integral de infancia, así como el cumplimiento de los requisitos que conlleva el reconocimiento CAI, requiere de conocimientos y competencias específicas por parte de quienes estén encargados de ponerlo en marcha. En 2015 se inicia el máster en Derechos y Políticas Municipales de Infancia, que surge de la colaboración del UCE con la AACID y la UPO. La formación en línea permitió que profesionales de diferentes países pudieran acceder a él. La utilización de una metodología docente basada en el estudio de caso y la resolución de problemas (Stoll y Ramil, 2014) incentivó la participación bajo la orientación del profesorado. Tras dos ediciones (2015-2016 y 2016-2017) se evaluó su impacto.

\subsection{Evaluación del impacto del máster en Derechos y Políticas de Infancia}

El objetivo del máster era dotar de una serie de herramientas — conceptuales y procedimentales - a profesionales — técnicos o políticos - que diseñaran e implementaran políticas municipales de infancia, proporcionándoles contenidos de dos tipos: los relacionados con las necesidades y los derechos de los NNA y los mecanismos de seguimiento y supervisión de su cumplimiento, además de los orientados al diseño integral de políticas municipales de infancia y la evaluación de su impacto.

El enfoque de EpD implica conocer qué incidencia tienen las competencias adquiridas sobre el contexto que queremos transformar, entendiendo que el «impacto es el efecto que la formación genera en una organización, como consecuencia de las competencias adquiridas" (Pineda, 2000, p. 124). Por ello la evaluación ha de ser una parte integral del proceso de cambio organizacional que queremos implementar (Tejada y Ferrández, 2007). Desde este enfoque (Fernández-Ferrer y Forés, 2018) se elaboró el Plan de Evaluación del Máster, cuyo objetivo final es la mejora del programa CAI y, con ello, de la garantía de los derechos de la infancia en el ámbito municipal.

Dada la extensión de este artículo solo se presentarán algunos resultados parciales relativos al impacto, entendido como los cambios que los aprendizajes generan en la persona formada, en la organización a la que pertenece e incluso, directa o indirectamente, en el contexto en el que se desarrollan sus acciones.

\subsubsection{Materiales y métodos}

La literatura ofrece numerosos modelos de evaluación de impacto (Kirkpatrick, 1959, 1996, 1999; Wade, 1994; Pineda, 2000). En este trabajo, siguiendo a Biencinto y Carballo (2004) se han establecido tres indicadores: 
- I1. Reacción. Percepción del alumnado sobre la formación recibida.

- I2. Aprendizaje. Alcance de los objetivos educativos y adquisición de competencias.

— I3. Impacto. Efectos de la formación en el individuo, la organización y el contexto.

Para la obtención de resultados se elaboró el siguiente sistema categorial (tabla 1).

Tabla 1. Categorización del impacto de la formación

\begin{tabular}{l}
\hline \multicolumn{1}{c}{ D1. Transferencia } \\
\hline C1. Cumplimiento de objetivos. \\
C2. Contribuciones al conocimiento nuevo. \\
C3. Contribuciones a la actualización y/o a la profundización. \\
C4. Contribuciones a la producción.
\end{tabular}

C4. Contribuciones a la producción.

\begin{tabular}{|c|c|}
\hline \multicolumn{2}{|c|}{ D2. Impacto en el sujeto } \\
\hline C5. Efectos: desempeño profesional. & $\begin{array}{l}\text { 5.1. Promoción de cargos con mayor responsabilidad. } \\
\text { 5.2. Participación como experto en eventos (foros, } \\
\text { conferencias, etc.). }\end{array}$ \\
\hline
\end{tabular}

C6. Efectos: producción intelectual.

C7. Efectos: consideración y/o reconocimiento.

\begin{tabular}{ll}
\hline & D3. \\
\hline C8. Contribuciones a la organización. & 8.1. Aumento de la productividad. \\
& 8.2. Reducción de costos. \\
& 8.3. Reducción de tiempos. \\
& 8.4. Mejora de la calidad. \\
& 8.5. Mejora del clima laboral. \\
8.6. Mejora de la motivación personal. & 8.7. Mejora del trabajo en equipo.
\end{tabular}

C9. Posibilidades de innovar o realizar cambios.

\section{D4. Impacto en el contexto social}

C10. Necesidades sociales que se cubren.

C11. Niveles de aplicación en el contexto de estrategias y conocimientos adquiridos.

\section{D5. Satisfacción a medio plazo}

C12. Satisfacción general.

C13. Cumplimiento de las expectativas.

C14. Recomendación a otros profesionales.

Fuente: elaboración propia a partir de Ramos, Meizoso y Guerra (2016).

Se diseñó un instrumento cuantitativo basado en una escala de 1 a 5 que constaba de 24 ítems distribuidos en 5 dimensiones: Datos generales, Aprendizaje, Impacto en el sujeto, Impacto en la organización e Impacto social. El cuestionario fue validado por expertos mediante la técnica Delphi y pilotado por un grupo de estudiantes. Se puso en Google Drive a disposición de los 49 estudiantes egresados del máster en las dos ediciones en el siguiente enlace: $<$ https://forms.gle/Zy3yvb8K2ciDzQqh6>. 
El análisis de resultados se ha abordado de manera global, basado en la triangulación de los datos obtenidos, tanto del proceso como de los resultados del aprendizaje: informes de evaluación, seguimiento y cuestionarios de satisfacción del alumnado.

\subsubsection{Resultados}

En este apartado se exponen los resultados más relevantes de cada indicador de manera integrada, ya que su interpretación adquiere mayor significado en el análisis combinado. La evaluación de impacto sigue en curso en el momento de realizar este trabajo, por lo que los datos presentados deben ser considerados como un avance, ya que representan el $47 \%$ del total del alumnado.

\section{I1. Reacción}

Según los resultados del cuestionario de satisfacción (tabla 2), se puede afirmar que la mayoría expresó una reacción muy positiva al programa en ambas ediciones.

Tabla 2. Distribución de las puntuaciones promedio de satisfacción

\begin{tabular}{lcccccc}
\hline Dimensiones & M 1Ed & DEI 1Ed & DEII 1Ed & M 2Ed & DEI 2Ed & DEII 2Ed \\
\hline $\begin{array}{l}\text { Objetivos y contenidos } \\
\text { (I 1-3) }\end{array}$ & 8,43 & 9,14 & 7,88 & 7,22 & 8,38 & 7,39 \\
\hline $\begin{array}{l}\text { Metodología } \\
\text { (I 4-6) }\end{array}$ & 8,50 & 8,00 & 8,13 & 8,00 & 7,44 & 7,67 \\
\hline $\begin{array}{l}\text { Docencia (I 7-10) } \\
\text { Medios }\end{array}$ & 9,10 & 9,36 & 8,63 & 8,42 & 8,06 & 8,08 \\
(I 11-17) & 8,47 & 8,48 & 8,29 & 8,33 & 7,63 & 8,25 \\
Utilidad (I 19-19) & 8,95 & 8,93 & 9,00 & 8,17 & 8,00 & 8,42 \\
\hline Resumen (I 21) & 8,70 & 8,86 & 7,75 & 7,70 & 8,13 & 7,33 \\
\hline
\end{tabular}

Fuente: extraído de García, López y Martínez (2020).

Utilidad de la formación junto a Docencia son los ámbitos en los que las puntuaciones permanecen más altas. Ambas se mantienen por encima de 8 puntos. Este resultado se corrobora con la aplicación del instrumento de evaluación de impacto (gráfico 1), en el que el alumnado mantiene los niveles de satisfacción. El $80 \%$ afirma que el programa ha cubierto sus expectativas.

El 91,3\% de encuestados declara que recomendaría el programa a otros profesionales (gráfico 2).

Otro aspecto a destacar es la flexibilidad de la formación en línea, que posibilita compatibilizar los estudios con la vida laboral.

[...] me lancé con el primer diploma, cuando vi la flexibilidad que ofrecía decidí cursar el segundo diploma y ahora podré obtener un título oficial de máster. (Periodista especialista en comunicación para organizaciones sin ánimo de lucro, ALM5DFLETFM) 
Gráfico 1. Cumplimiento de expectativas

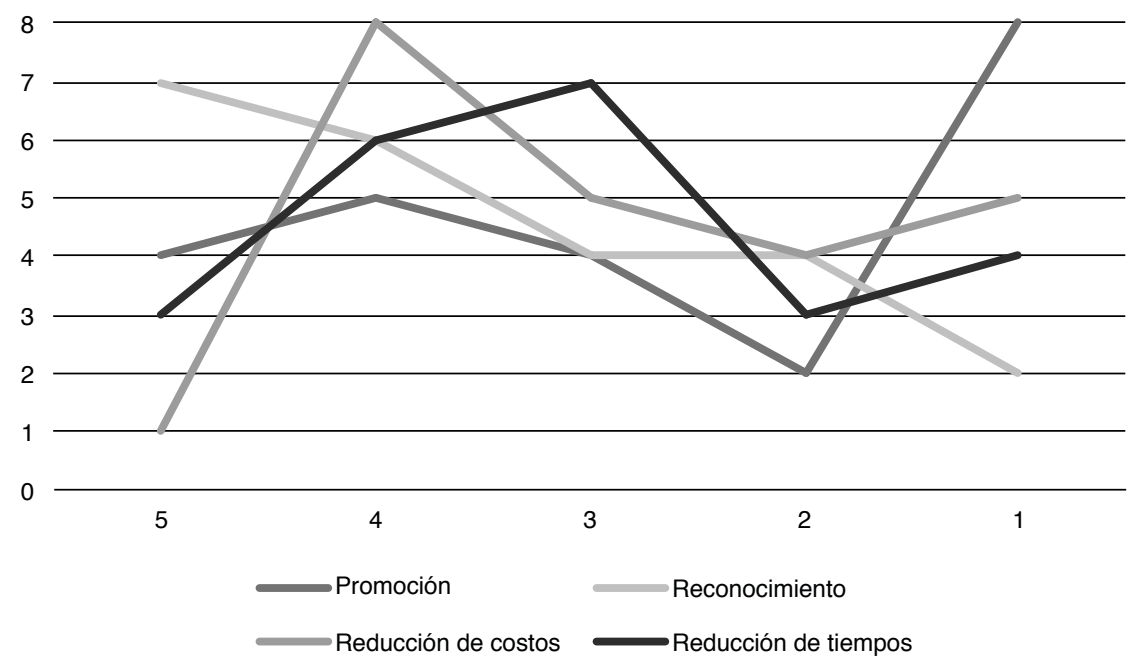

Fuente: elaboración propia.

Gráfico 2. Recomendación de la formación a otros profesionales

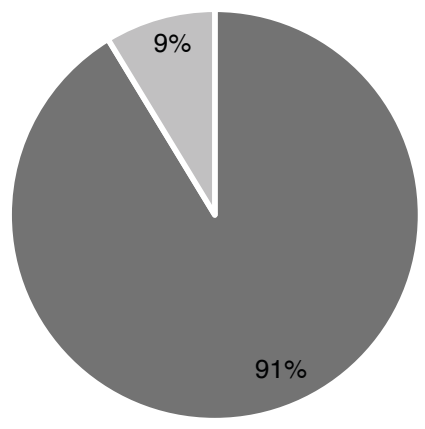

Sí $\quad$ No

Fuente: elaboración propia.

La valoración positiva del alumnado sobre los diferentes elementos del programa se mantiene en el tiempo, lo que da consistencia al indicador. 


\section{I2. Aprendizaje}

Más del 95\% de los sujetos puntúan positivamente el grado de cumplimiento de los objetivos (gráfico 3), lo que complementa que uno de los aspectos más valorados sea la utilidad práctica de la formación (tabla 2).

Tanto el nivel de aprendizaje aplicado (gráfico 4) como la mejora de los niveles de producción tras cursar el programa son muy positivos.

En ambos ítems las puntuaciones se concentran en los niveles más altos de la escala. El 95,6\% considera que el programa le ha ayudado a actualizar y profundizar en derechos y políticas de infancia, y el $86,9 \%$ afirma haber mejorado sus niveles de producción de materiales, estrategias y proyectos de participación infantil.

Gráfico 3. Grado de cumplimiento de objetivos

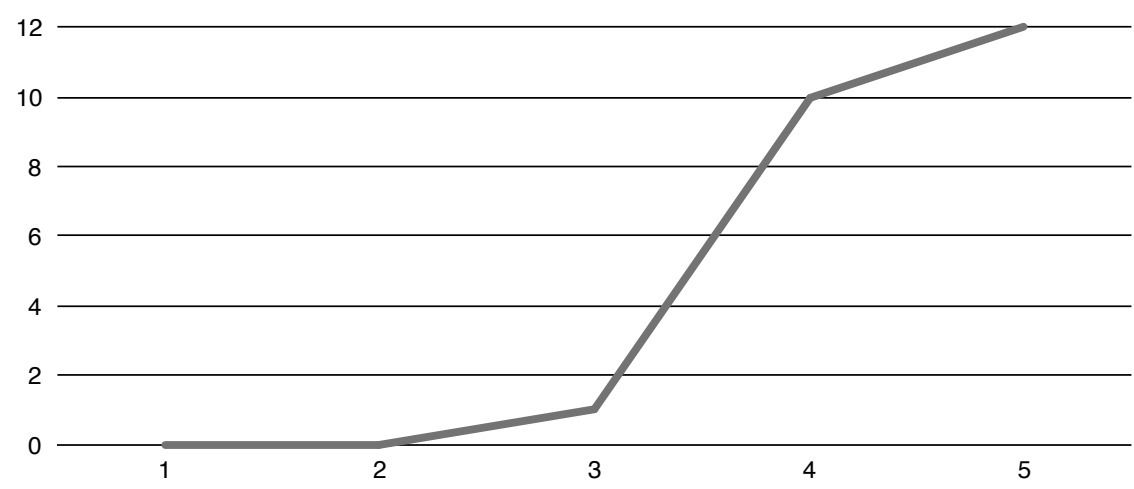

Fuente: elaboración propia.

Gráfico 4. Niveles de aprendizaje aplicado

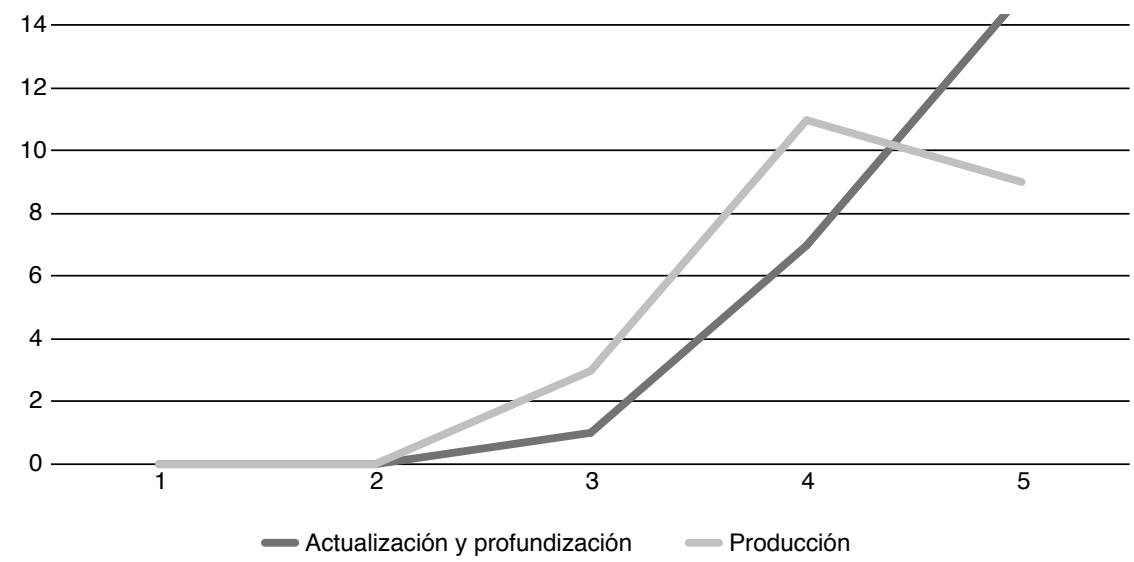

Fuente: elaboración propia. 
Complementando esta información con el análisis del contenido de actividades, foros y trabajos fin de máster (TFM), se constata el valor de las competencias adquiridas para la mejora de su realización profesional.

[...] el temario me ha permitido sistematizar y priorizar mi experiencia como coordinadora de programas de participación infantil y adolescente. (Animadora sociocultural de ayuntamiento, ALM1DTRATFM)

Igualmente, se destaca la oportunidad de completar sus conocimientos con un máster de estas características y obtener una titulación de postgrado.

Solo desde la profesionalización podemos desarrollar un trabajo de calidad. (Educador social de un instituto provincial, ALM2DTRATFM)

\section{I3. Impacto}

La formación tiene una incidencia positiva sobre la estabilidad laboral (gráfico 5). Aumenta en un $13 \%$ el número de sujetos que consigue un contrato indefinido después de realizar el máster y disminuye en un 17,4\% el de los que tienen un contrato "por obra y servicio». Un $4,4 \%$ de los egresados se integra en un campo laboral diferente.

Gráfico 5. Situación laboral anterior y posterior a la formación

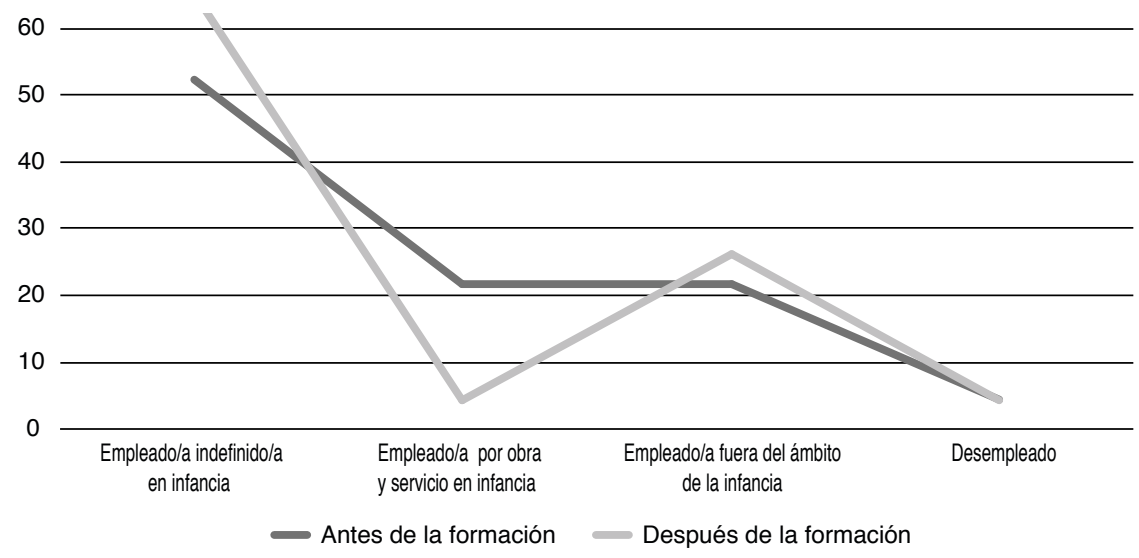

Fuente: elaboración propia.

La percepción sobre la mejora en sus competencias laborales resulta positiva (gráfico 6). Un 74\% afirma que tras cursar el máster ha aumentado la calidad de su trabajo.

Un $52,1 \%$ está de acuerdo en que las competencias adquiridas contribuyen a mejorar su productividad y un $65,2 \%$ considera que han aumentado sus posibilidades de innovar en el trabajo. 
Gráfico 6. Impacto en el sujeto

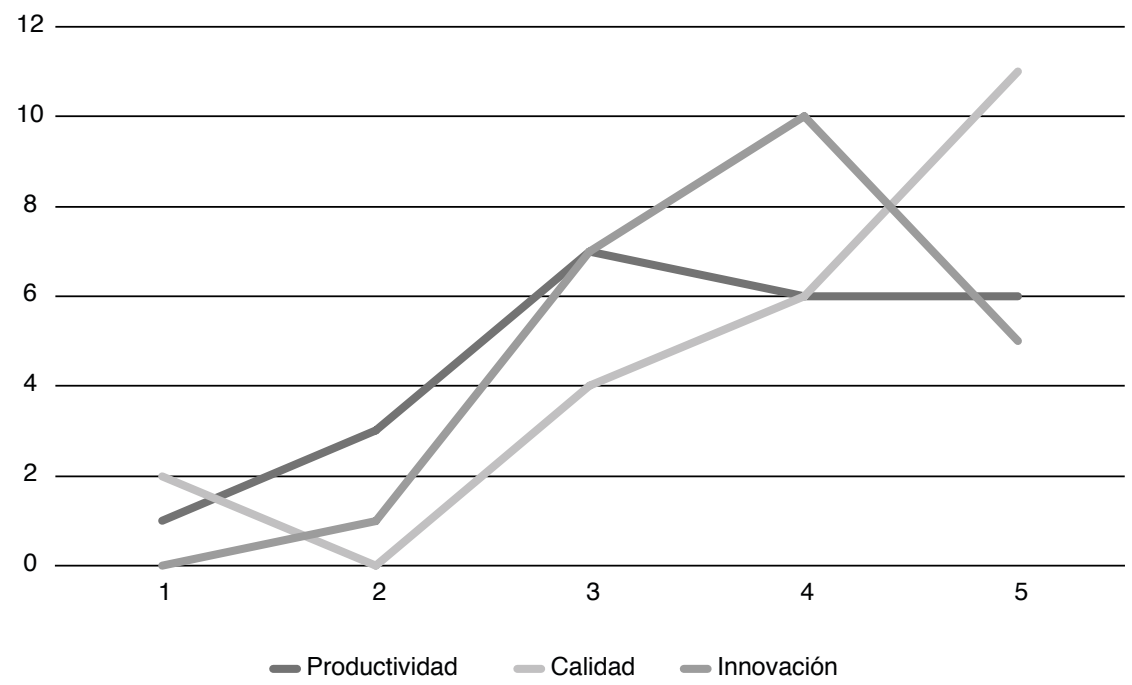

Fuente: elaboración propia.

Gráfico 7. Impacto en las organizaciones

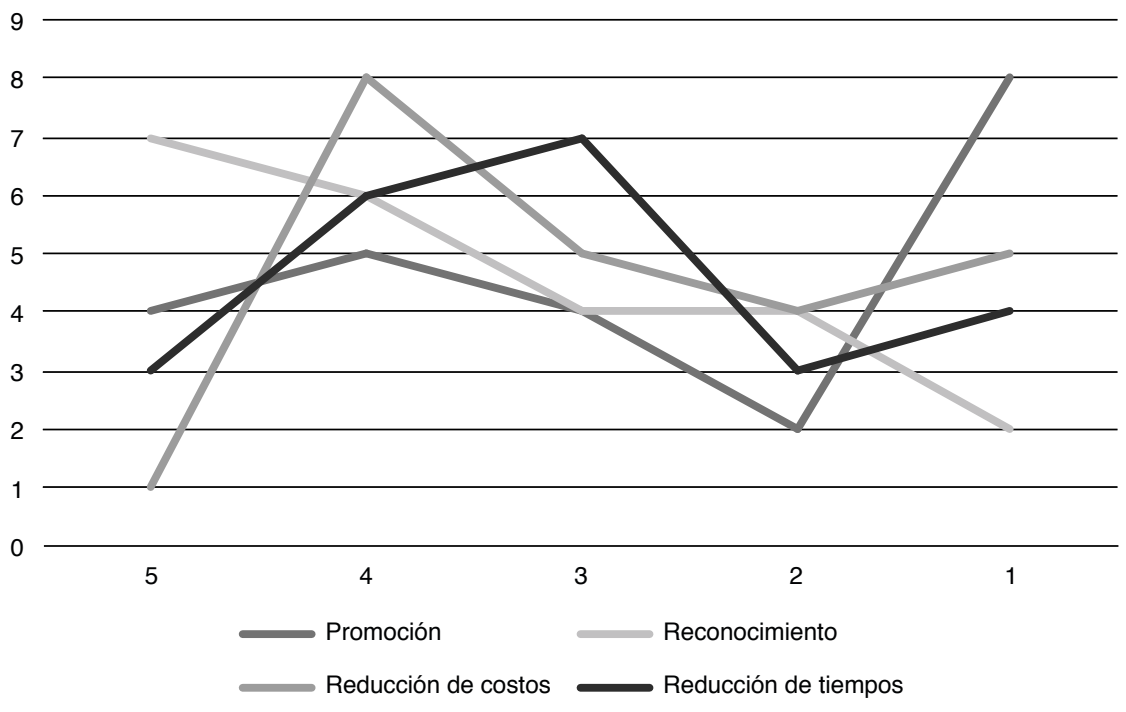

Fuente: elaboración propia.

Los resultados referidos al impacto de la formación en la organización no son tan positivos. El 34\% de los egresados no promocionaron tras acabar el 
máster. Tampoco encontramos un porcentaje relevante de sujetos que se sientan más reconocidos una vez concluida la formación (gráfico 7).

Así, los egresados perciben mejoras en sus capacidades laborales, pero dichas mejoras no son reconocidas ni valoradas en las organizaciones.

Los resultados del impacto social del máster son muy positivos (gráfico 8). El 73,9\% afirma aplicar las estrategias y los recursos aprendidos en el contexto en el que desarrolla su actividad profesional. Un $65,2 \%$ señala que lo que aprendió le resulta de gran ayuda para satisfacer las necesidades de los NNA, así como para trabajar en contextos de inclusión y empoderamiento infantil.

Gráfico 8. Impacto social

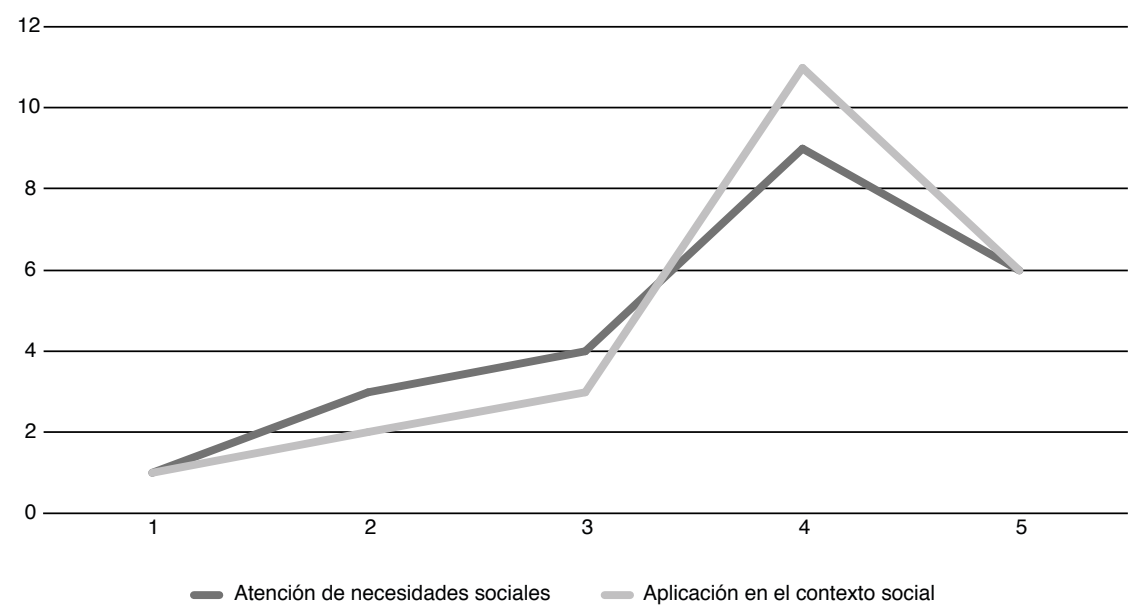

Fuente: elaboración propia.

La aplicación de la CDN, la colaboración con ayuntamientos y la visibilización de las problemáticas de los NNA son algunas de las respuestas obtenidas por las personas encuestadas al preguntarles qué tareas o acciones ven mejoradas tras adquirir las nuevas competencias.

\section{Conclusiones}

La Agenda 2030 nos compromete en la consecución de una sociedad más justa, equitativa, inclusiva y sostenible, en la que los derechos humanos se conviertan en una realidad para todos, con independencia de cualquier otra consideración. La forma de conceptualizar el desarrollo que subyace a los ODS establecidos en la Agenda hace que los derechos humanos se conviertan en un eje transversal de la EpD. Este enfoque, centrado en el desarrollo humano, empodera a las personas para que exijan el cumplimiento de los derechos que aparecen recogidos en los tratados internacionales, ejerciendo a través de la 
participación todos sus derechos de ciudadanía. Cuando nos referimos a los derechos de ciudadanía de los más jóvenes, el tratado internacional que nos sirve como fundamento moral es la CDN, ya que solo será posible alcanzar los ODS si se apuesta por un modelo social, político y económico en el que la infancia sea una prioridad, adoptando medidas especiales para aquella que se encuentra en situación de mayor vulnerabilidad. Por tanto, es necesario adoptar el enfoque de derechos de la infancia en la EpD.

En España el UCE ha liderado, desde la década de 1990, el trabajo en EpD con un enfoque de derechos de la infancia a través de distintas iniciativas llevadas a cabo siempre en colaboración con diferentes aliados. Se han desarrollado programas de sensibilización en contextos educativos formales, líneas de investigación que han permitido tener mayor conocimiento sobre la situación de la infancia y programas formativos en colaboración con universidades que han capacitado a un gran número de profesionales que, en la actualidad, desempeñan su actividad laboral en diferentes niveles de la Administración, desarrollando políticas públicas de infancia de ámbito local, autonómico y/o estatal.

Una de las experiencias más exitosas del UCE en la que participan aliados procedentes de distintos ámbitos de la Administración pública es el programa CAI, derivado de la iniciativa de UNICEF Internacional «Child Friendly Cities». Una CAI es un municipio que garantiza el bienestar infantil adoptando políticas integrales de infancia que satisfacen sus necesidades y dan respuestas adecuadas al ejercicio de sus derechos, al tiempo que permiten que los NNA adopten un papel activo en la toma de decisiones que les afectan. La consecución del reconocimiento de ciudad amiga de la infancia exige de unas competencias profesionales de las que deben disponer los responsables - políticos y/o técnicos- de los servicios, de los programas y de las medidas destinadas a mejorar la situación de los NNA en el municipio.

El máster en Derechos y Políticas Municipales de Infancia, diseñado para dotar a los profesionales de estas competencias, ha permitido obtener un reconocimiento académico y profesional a un grupo de personas que han transformado los entornos en los que se produce la socialización de los NNA. La evaluación de impacto de este máster, como una de las fases del mismo (Fernández-Ferrer y Forés, 2018; Tejada y Ferrández 2007), pone de manifiesto la importancia que ha tenido para actualizar y profundizar sus conocimientos, lo que les ha permitido mejorar tanto sus competencias profesionales como la calidad de su trabajo, al mismo tiempo que han sido capaces de desarrollar estrategias integrales de infancia orientadas a mejorar el bienestar de los NNA. Por el contrario, el impacto de la formación no se ha visto reflejado de manera tan positiva en la consideración y el reconocimiento que estos profesionales reciben por parte de las organizaciones en las que están desarrollando su labor.

El plan de evaluación del máster nos permite concluir sobre la posibilidad de transformar contextos a partir de la aplicación de una serie de competencias adquiridas (Pineda, 2000), lo que implica la importancia de la formación para dotar a los profesionales de la EpD de un sustento teórico y de una serie de herramientas prácticas que den soporte a su trabajo. Asimismo, se destaca la 
necesidad de formar no solo desde las instituciones, sino también para las instituciones - estableciendo redes, compartiendo experiencias y evaluando impactos a nivel global—, ya que solo de ese modo será posible asumir el reto y afrontar el compromiso que supone la Agenda 2030.

\section{Referencias bibliográficas}

Biencinto, C. y Carballo, R. (2004). Revisión de modelos de evaluación del impacto de la formación en el ámbito sanitario: De lo general a lo específico. Revista Electrónica de Investigación y Evaluación Educativa, 10(2), 101-116. $<$ https://doi.org/10.7203/relieve.10.2.4324>

CONGD (2005). Educación para el Desarrollo: Una estrategia de cooperación imprescindible. Madrid: CONGD.

Cornia, A. y Jolly, R. (1987). Ajuste con rostro humano. Madrid: UNICEF / Siglo XXI.

FernándeZ-Ferrer, M. y Forés, A. (2018). Evaluación del desarrollo competencial en la educación superior: La perspectiva del profesorado universitario. Educar, 54(2), 391-410. <https://doi.org/10.5565/rev/educar.799>

FonTaINE, S. (1995). Education for Development: A teacher's resource for global learning. Nueva York: UNICEF-Hodder \& Stoughton.

García Pérez, R.; López Catalán, L. y Martínez Gimeno, A. (2020). Defending children's rights at the local level: An innovative practice in the formation of leaders in favor of children. Revista IJERI, 13. En prensa. Fecha de aprobación 21 de enero de 2019.

Kirkpatrick, D.L. (1959). Evaluating Training Programs. San Francisco: Berrett Koehler.

- (1996). Great ideas revisited. Training and Development, 50(1), 54- 65.

- (1999). Evaluación de acciones formativas: Los cuatro niveles. Barcelona: Gestión 2000.

Ministerio de Asuntos Exteriores y de Cooperación (2007). Estrategia de la Educación para el Desarrollo de la Cooperación Española. Madrid: Secretaría de Estado de Cooperación Internacional. Recuperado de $<$ http://intercoonecta.aecid. es/Documentos\%20de\%20la\%20comunidad/Estrategia_Educación\%20para\%20 Desarrollo.pdf>.

Pineda, P. (2000). Evaluación del impacto de la formación en las organizaciones. Educar, 27, 119-133. $<$ https://doi.org/10.5565/rev/educar.253>.

PNUD (1990). Desarrollo humano: Informe 1990. Bogotá: Tercer Mundo Editores.

Ramos, F.; Meizoso, M.C. y Guerra, R.M. (2016). Instrumento para la evaluación del impacto de la formación académica. Revista Universidad y Sociedad, 8(2), 114124.

Sen, A. (2000). Desarrollo y libertad. Buenos Aires: Planeta.

Stoll, L. y Ramil, X. (2014). Metodología para el desarrollo de estudios de caso. Madrid: UPM.

Tejada, J. y Ferrández, E. (2007). La evaluación del impacto de la formación como estrategia de mejora en las organizaciones. Revista Electrónica de Investigación Educativa, 9(2). Recuperado de <https://redie.uabc.mx/redie/article/view/167>. 
UNGD Human Rights Working Group (2003). The human rigths based approach to development cooperation towards a common understanding among. Nueva York: United Nations.

UNICEF (1978). Educación para el Desarrollo. Madrid: UNICEF-ANELE.

- (1987). Ajuste con rostro humano. Nueva York: UNICEF.

- (1996a). Informe de la Junta Ejecutiva del Fondo de Naciones Unidas para la Infancia: Documentos Oficiales. Suplemento n. ${ }^{\circ}$ 12. Madrid: Consejo Económico y Social. Recuperado de <https://www.unicef.org/about/execboard/files/1996-12_ Rev.1_Report-ODS-Spanish.pdf>.

- (1996b). Manual para charlas en las escuelas. Madrid: UCE.

- (2007). Un mundo apropiado para nosotros. UNICEF NY noviembre de 2007. Recuperado de <https://www.unicef.org/spanish/publications/index_42122. html>.

- (2010). Progreso para la infancia: Lograr los ODM con equidad. Nueva York: UNICEF.

- (2017). Construir el futuro: Los niños y los objetivos de desarrollo sostenible en los países ricos. Innocentti Report Card n. ${ }^{\circ} 14$. Florencia: Innocentti Research Center. Recuperado de <https://www.unicef-irc.org/publications/892-construirel-futuro-los-ni\%C3\%B1 os-y-los-objetivos-de-desarrollo-sostenible-en-lospa\%C3\%ADses.html>

- (2018a). Participación infantil en los centros escolares. Madrid: UCE.

- (2018b). UNICEF Child Friendly Cities and Communities: Hadnbook. Recuperado de <https://www.unicef.org/eap/sites/unicef.org.eap/files/2018-04/unicef-childfriendly-cities-and-communities-handbook.pdfs

Wade, P. (1994). Measuring the Impact of Training. Londres: Kogan Page. 